\title{
Celiac disease in the East and the West: Bridging the gaps between the guidelines and their implementation in daily practice is mandatory
}

\author{
Anmol Dhawan $^{1} \cdot$ Ashish Agarwal $^{1} \cdot$ Chris J. Mulder ${ }^{2} \cdot$ Govind K. Makharia $^{1}$ \\ Published online: 17 July 2019 \\ (C) Indian Society of Gastroenterology 2019
}

\section{Introduction}

Recently, two questionnaire-based surveys of pediatricians, one from UK by Paul et al. and another by Malik et al. from India, have been published in the Indian Journal of Gastroenterology, which highlight the pattern of current practices in the diagnosis and management of patients with celiac disease (CeD) [1,2]. While Paul et al. [1] utilized either a telephone or an electronic mail to conduct their survey involving 100 pediatricians practicing in Southwestern England, Malik et al. [2] used electronic mail alone involving 271 pediatricians across India. Paul et al. focused primarily on the awareness of pediatricians about European Society of Paediatric Gastroenterology Hepatology and Nutrition (ESPGHAN) guideline of the non-biopsy pathway for the diagnosis of CeD [1]. Malik et al., on the other hand, focused on the overall practice pattern pertaining to $\mathrm{CeD}$ such as its most common presenting symptoms, known associations, standard diagnostic criteria, and its management [2]. These two studies have not only brought a few interesting observations but also a few alarming patterns of practices both for the diagnosis and the management of $\mathrm{CeD}[1,2]$. We have selected a few relevant points from both the studies, and we have reviewed the standard guidelines and the relevant literature around these points and summarized these in this technical report.

Govind K. Makharia

govindmakharia@gmail.com

1 Department of Gastroenterology and Human Nutrition, All India Institute of Medical Sciences, New Delhi 110 029, India

2 Department of Gastroenterology, Amsterdam University Medical Centre, Amsterdam, The Netherlands

\section{Burden of the disease}

Until a few decades ago, CeD has been considered to be an uncommon disorder affecting mainly children and remained limited to the Western Europe [3, 4]. However, over time, it was realized that there is no age bar for $\mathrm{CeD}$ as it can affect any age group; moreover, it is a global disease. Recognition of the wide spectrum of $\mathrm{CeD}$, simplification of the diagnostic criteria, and widespread use of celiac-specific serological tests (antitissue transglutaminase [tTG] antibody, anti-endomysial antibody [AEA], or anti-deamidated gliadin peptide antibody) have led to an increase in the recognition of CeD globally [3, 4].

Population-based screening studies from Europe, North America, and other parts of the world indicate that $\mathrm{CeD}$ is a major public health problem [5-8]. We, in a systematic review and meta-analysis of these studies, showed that the pooled global seroprevalence of $\mathrm{CeD}$ in the general population is $1.4 \%$ (95\% confidence interval [CI] 1.1 to $1.7 \%$ ) [9]. Most population-based epidemiological studies on $\mathrm{CeD}$ prevalence are based on serological data, and the diagnosis of $\mathrm{CeD}$ in all seropositive patients has not been confirmed by invasive small-intestinal mucosal biopsies. Therefore, the global pooled prevalence of biopsy-proven $\mathrm{CeD}$, which is $0.7 \%$ ( $95 \%$ CI 0.5 to $0.9 \%$ ), is lower than the seroprevalence of $\mathrm{CeD}$. Based on these data, approximately 37 to 59 million people are expected to suffer from $\mathrm{CeD}$ worldwide. While $\mathrm{CeD}$ is still evolving in many countries, interestingly, the prevalence of $\mathrm{CeD}$ has increased by 2 -fold over the last two decades in many European countries and USA $[8,10]$. Of the world's top ten most populated countries, population-based prevalence data on $\mathrm{CeD}$ are available from India, the US, Brazil, and Russia but are largely lacking from China, Indonesia, Pakistan, Nigeria, Bangladesh, and Japan [9].

Traditionally, $\mathrm{CeD}$ was thought to be uncommon in India; however, an increase in the number of cases had been 
recognized in late 1990 and 2000 onwards [11-16]. Three population-based studies, two from the northern parts of India and another multi-centric pan Indian study, showed that the overall prevalence of $\mathrm{CeD}$ in India is $0.67 \%$ and $1.2 \%$ in northern parts of India [17-19]. These estimates suggest that there are 6 to 8 million patients with CeD in India, and of that, only a minority are actually diagnosed or come to clinicians' attention.

While the global pool of the patients is so large, most patients (83\% and 95\%) in developed countries, and possibly an even higher number in developing countries, still remain undiagnosed $[3,4,9]$. This large pool remains unrecognized partly because of lack of classical gastrointestinal (GI) symptoms in approximately half of the patients. The spectrum of clinical manifestations of $\mathrm{CeD}$ is wide and includes both GI symptoms such as chronic diarrhea and dyspepsia, and extraGI manifestations such as short stature, failure to thrive, anemia, dermatitis herpetiformis, infertility, and liver diseases $[20,21]$.

While there was a poor awareness about $\mathrm{CeD}$ not only among physicians but also general public, the survey by Malik et al. [2] showed that awareness has increased not only in them but also among the parents of children with $\mathrm{CeD}$ and that has led to an increased detection of $\mathrm{CeD}$ [2]. The increase in the awareness about $\mathrm{CeD}$ is also reflected by increasing number of scientific publications, establishment of patient support groups and celiac disease societies, discussions about $\mathrm{CeD}$ in medical conferences and continuing medical education (CME) programs, increase in the market value of glutenfree products, and a distinct increase in the media coverage about $\mathrm{CeD}$ and gluten-related disorders.

\section{Evolution of the diagnostic criteria and challenges in their implementation in the diagnosis of $\mathrm{CeD}$}

The diagnostic criteria for $\mathrm{CeD}$ have evolved over time. The first criterion for the diagnosis of CeD was laid down in 1979. The Interlaken guidelines was based on the outcome of three sequential intestinal biopsies, i.e. demonstration of villous abnormalities on a normal gluten-containing diet, resolution of these abnormalities on gluten-free diet (GFD), and their recurrence on reintroduction of gluten-containing diet [22]. During that time, only a limited number of patients could be recognized to have $\mathrm{CeD}$ because of rigor of the diagnostic criteria, which could be followed in general practice.

In 1990, the ESPGHAN issued revised diagnostic criteria from the requirement of three sequential intestinal biopsies to just one at the beginning to demonstrate villous atrophy for the diagnosis of $\mathrm{CeD}$ in patients greater than 2 years of age; however, for confirmation of the diagnosis, a persistent response to GFD after $2-$ 3 months was required [23].

The foundation of modern-day serological tests was laid in 1970s when Shiner and Ballard showed an increase in extracellular deposits of immunoglobulins, especially $\operatorname{IgA}$, in the jejunal mucosa of children with $\mathrm{CeD}$ after a gluten challenge [24, 25]. Immediately thereafter, similar IgA deposits in the basement membrane were also reported in the small-intestinal mucosa of untreated patients with $\mathrm{CeD}[26,27]$. It was also found that during a GFD, the antibody deposits disappeared, but when gluten was reintroduced in their diet, the antibody deposition rapidly reappeared in them [28]. Later, anti-gliadin antibody (AGA) was described and AGA remained the first line celiac-specific serological test until the 1990s [29]. In 1990s, AEA was discovered and a combination of AGA with AEA testing became the standard diagnostic strategy [30]. Subsequently, a high false positive rate for AGA was observed, which led to a fall in the use of AGA in the screening of patients suspected to have CeD. Later, with the discovery of anti-tTG as a substrate for AEA, tTG-based enzyme-linked immunoassays (ELISA) became the standard diagnostic test for $\mathrm{CeD}$ [31].

Of all the serological tests, IgA anti-tTG2 antibody is the most widely used test both for the diagnosis and initial screening for $\mathrm{CeD}$ because of its high sensitivity and specificity, ease of use, and its quantitative capability. In a recent systematic review, Chou et al. reported a pooled sensitivity of anti-tTG antibody to be $92.8 \%$ ( $95 \%$ CI 90.3 to $94.8 \%$ ); specificity $97.9 \%$ (95\% CI 96.4 to $98.8 \%$ ); a positive likelihood ratio (LR) of 45.1 (95\% CI 25.1 to 75.5$)$ and negative LR of 0.07 (95\% CI 0.05 to 0.10 ) [32]. IgA anti-tTG antibody levels also correlate with the degree of severity of mucosal damage, and a titer of 10-fold or higher over the upper limit of normal (ULN) predicts the presence of villous abnormality with very high specificity [33].

With the above-mentioned observations, in 2012, ESPGHAN came out with a revised set of guidelines, according to which the need for a mucosal biopsy can be obviated in symptomatic children if (a) levels of IgA anti-tTG antibody are elevated to more than ten times the ULN; (b) if the patient is positive for AEA; and (c) if the patient is positive for HLA-DQ2/DQ8 [34]. While the ESPGHAN suggests a non-biopsy pathway of diagnosis, most other guidelines including American College of Gastroenterology, British Society of Gastroenterology, European Society for the Study of Coeliac Disease, World Gastroenterology Organization, and Indian guidelines suggest that demonstration of significant villous abnormality on duodenal mucosal biopsies is essential for the diagnosis of $\mathrm{CeD}$ [34-39]. 


\section{The basis for a non-biopsy approach for the diagnosis of $\mathrm{CeD}$}

The pathological changes in patients with celiac autoimmunity and $\mathrm{CeD}$ are a continuum from raised intraepithelial lymphocytes to crypt hypertrophy to varying degree of villous abnormalities. At present, the diagnosis of $\mathrm{CeD}$ is made on the basis of presence of villous abnormalities of modified Marsh grade 2 and above. The relevant question is whether we can predict the presence of villous abnormalities based on the titer of anti-tTG antibody in the serum? There are studies to suggest that presence of significant villous abnormalities can be predicted with high degree of sensitivity and specificity if the anti-tTG titer is more than 10-fold ULN and such a consistent observation forms the basis for the non-biopsy approach of the recent ESPGHAN criteria for the diagnosis of $\mathrm{CeD}$. The reliability of serological tests provided credence to ESPGHAN to recommend that in a subset of symptomatic patients, the diagnosis of $\mathrm{CeD}$ may be established using antitTG antibody levels 10-fold the ULN along with positive AEA in the presence of HLA compatible with $\mathrm{CeD}$, thus obviating the need for duodenal biopsy [34].

While there are well-defined guidelines for the diagnosis of CeD with both biopsy and non-biopsy approaches, the awareness and uptake of guidelines by pediatricians and physicians is not optimal. The study by Paul et al. [1] was primarily aimed at assessing awareness of the non-biopsy pathway of the ESPGHAN 2012 criteria among pediatricians practicing in Southwestern England. While 100 of 101 respondents were aware of the existence of such criteria, only 17 respondents stated that all the criteria were required for the diagnosis by a non-biopsy pathway, and 33 of them would make a diagnosis based on the anti-tTG titer alone even in asymptomatic children [1]. The study from India by Malik et al. [2] reflects the current practice pattern in India and the data is alarming. First of all, only half of the pediatricians would follow the laid down guidelines, while $26.5 \%$ would make a diagnosis of CeD based on a single value of anti-tTG antibody and $6.8 \%$ with a positive AEA alone. Furthermore, $10.3 \%$ of them would make the diagnosis of $\mathrm{CeD}$ based on a positive AGA, which is no more recommended either as a screening or a diagnostic serological test for $\mathrm{CeD}$ because of a high false positivity rate. Since $\mathrm{CeD}$ is a lifelong disease, all attempts should be made to make a definitive diagnosis based on the laid down criteria. A diagnosis based on insufficient evidence is likely to lead to challenges in near future, especially once there is a poor response to GFD. One will not be able to differentiate if the non-response to GFD is because of poor adherence to it or a wrong diagnosis. We would like to emphasize that both the serological tests and the histological changes will revert with GFD and hence a normal serology and a normal biopsy later on during follow up will neither confirm nor refute the diagnosis of CeD.

\section{Challenges associated with a diagnosis based on serology alone}

An anti-tTG antibody at a lower titer may not always reflect the presence of villous abnormalities and this may be a part of celiac autoimmunity and not CeD. Furthermore, there could be a false positive anti-tTG antibody in various other autoimmune diseases and chronic liver diseases. Human tTG antigens used in commercial kits are variable ranging from recombinant human $\mathrm{tTG}$ to human $\mathrm{TTG}$ cross-linked to gliadin specific peptides [40-42]. In addition, commercial kits typically provide sensitivity and specificity values that are calculated using small, poorly defined populations, which can be misleading. Several studies comparing different anti-tTG antibody-based assays from different manufacturers have revealed variable sensitivities and specificities for detecting $\mathrm{CeD}$; however, most of these studies were small in sample size and did not have the necessary power to accurately comment on the diagnostic accuracies of the testing $[40,43]$.

\section{Screening of the first-degree relatives of patients with CeD}

The first-degree relatives (FDR) of patients with $\mathrm{CeD}$, comprising of their parents, siblings, and children, are at approximately 7-8-fold higher risk of developing $\mathrm{CeD}$ as compared with the general population [44]. Among FDRs, sisters and daughters are at the highest risk (approximately 14-folds), in comparison with sons and brothers who are at 6-folds higher risk of developing $\mathrm{CeD}[45,46]$. While all the first-degree relatives of patients with $\mathrm{CeD}$ should be screened for it using anti-tTG antibody, only $46 \%$ of pediatricians in the study by Malik et al. and $45 \%$ of other healthcare professionals in one of our previous studies would like to screen them for $\mathrm{CeD}$ [47]. We identify this observation is also a gap in the appropriate management of patients with $\mathrm{CeD}$ and their families.

\section{Treatment of patients with CeD}

The standard of treatment of CeD at present time is lifelong and complete avoidance of cereals and food products containing gluten (GFD); in the study by Malik et al. [2], 12.3\% of pediatricians felt that gluten could be re-initiated after patients have improved on GFD. In a double-blind, placebo-controlled prospective study, Catassi et al. [48] demonstrated that an intake of as little as $50 \mathrm{mg}$ of gluten per day for 3 months was sufficient to cause a significant decrease in the intestinal mucosal villous height/crypt depth ratio. Therefore, a lifelong and complete avoidance of gluten should be maintained. All patients should be counseled both by the physicians and trained dieticians about GFD. While it is easy to prescribe 
GFD, it is extremely challenging for patients to adhere to the treatment completely because of widespread use of gluten in the food industry. Lapses in dietary adherence, inadvertent or advertent, have been reported in $42 \%$ and $96 \%$ of patients with $\mathrm{CeD}$ [49].

In conclusion, $\mathrm{CeD}$ is a worldwide public health problem with an estimated pool of 37 to 59 million patients globally of which only a small fraction has either been diagnosed or received clinical attention. While there were well laid down guidelines by various international and national societies for the diagnosis and management of $\mathrm{CeD}$, gaps in their appropriate implementation in the general practice appear alarming. We believe that the two recent surveys, as described above, are the wake-up call for increasing awareness among the pediatricians and physicians about appropriate implementation of the diagnostic algorithm [50]. CeD is a lifelong disease and complete avoidance of gluten-containing food products is the only known effective treatment for it. While it is easy to prescribe GFD to these patients, there are challenges in maintaining a good compliance to it. Therefore, it is essential that a reliable diagnosis of $\mathrm{CeD}$ is made before instituting GFD in these patients.

\section{Compliance with ethical standards}

Conflict of interest $\mathrm{AD}, \mathrm{AA}, \mathrm{CJM}$, and GKM declare that they have no conflict of interest.

Disclaimer The authors are solely responsible for the data and the content of the paper. In no way, the Honorary Editor-in-Chief, Editorial Board Members, or the printer/publishers are responsible for the results/ findings and content of this article.

\section{References}

1. Paul SP, Adams HL, Basude D, Collaborators. Interpretation and implementation of the revised European Society for Paediatric Gastroenterology Hepatology and Nutrition (ESPGHAN) guidelines on pediatric celiac disease amongst consultant general pediatricians in Southwest of England. Indian J Gastroenterol. 2019;38. https://doi.org/10.1007/s12664-019-00952-9.

2. Malik I, Kumar K, Hussain H, Bhatia V, Sibal A, Malhotra S. Celiac disease: what Indian pediatricians know about the disease. Indian J Gastroenterol. 2019;38. https://doi.org/10.1007/s12664019-00958-3.

3. Lindfors K, Ciacci C, Kurppa K, et al. Coeliac disease. Nat Rev Dis Primer. 2019;5:3.

4. Lebwohl B, Sanders DS, Green PHR. Coeliac disease. Lancet. 2018;391:70-81.

5. Catassi C, Rätsch IM, Fabiani E, et al. Coeliac disease in the year 2000: exploring the iceberg. Lancet. 1994;343:200-3.

6. Mäki M, Mustalahti K, Kokkonen J, et al. Prevalence of celiac disease among children in Finland. N Engl J Med. 2003;348: 2517-24.

7. Mustalahti K, Catassi C, Reunanen A, et al. The prevalence of celiac disease in Europe: results of a centralized, international mass screening project. Ann Med. 2010;42:587-95.
8. Lohi S, Mustalahti K, Kaukinen K, et al. Increasing prevalence of coeliac disease over time. Aliment Pharmacol Ther. 2007;26:121725 .

9. Singh P, Arora A, Strand TA, et al. Global prevalence of celiac disease: systematic review and meta-analysis. Clin Gastroenterol Hepatol. 2018;16:823-36.e2.

10. Catassi C, Kryszak D, Bhatti B, et al. Natural history of celiac disease autoimmunity in a USA cohort followed since 1974. Ann Med. 2010;42:530-8.

11. Yadav P, Das P, Mirdha BR, et al. Current spectrum of malabsorption syndrome in adults in India. Indian J Gastroenterol. 2011;30: 22-8.

12. Yachha SK, Poddar U. Celiac disease in India. Indian J Gastroenterol. 2007;26:230-7.

13. Sood A, Midha V, Sood N, Kaushal V, Puri H. Increasing incidence of celiac disease in India. Am J Gastroenterol. 2001;96:2804-5.

14. Nijhawan S, Katiyar P, Nagaich N, et al. Prevalence of associated disorders in Indian patients with celiac disease. Indian J Gastroenterol. 2013;32:330-4.

15. Kochhar R, Jain K, Thapa BR, et al. Clinical presentation of celiac disease among pediatric compared to adolescent and adult patients. Indian J Gastroenterol. 2012;31:116-20.

16. Sood A, Midha V, Sood N, Avasthi G, Sehgal A. Prevalence of celiac disease among school children in Punjab. North India J Gastroenterol Hepatol. 2006;21:1622-5.

17. Makharia GK, Verma AK, Amarchand R, et al. Prevalence of celiac disease in the northern part of India: a community based study. J Gastroenterol Hepatol. 2011;26:894-900.

18. Ramakrishna BS, Makharia GK, Chetri K, et al. Prevalence of adult celiac disease in India: regional variations and associations. Am J Gastroenterol. 2016;111:115-23.

19. Makharia GK, Baba CS, Khadgawat R, et al. Celiac disease: variations of presentations in adults. Indian J Gastroenterol. 2007;26: $162-6$.

20. Ludvigsson JF, Leffler DA, Bai JC, et al. The Oslo definitions for coeliac disease and related terms. Gut. 2013;62:43-52.

21. Das P, Makharia GK. Gut-liver axis and disease infidelity: a subject worth exploring. Indian J Gastroenterol. 2014;33:503-6.

22. McNeish AS, Harms HK, Rey J, Shmerling DH, Visakorpi JK, Walker-Smith JA. The diagnosis of coeliac disease. A commentary on the current practices of members of the European Society for Paediatric Gastroenterology and Nutrition (ESPGAN). Arch Dis Child. 1979;54:783-6.

23. Revised criteria for diagnosis of coeliac disease. Report of Working Group of European Society of Paediatric Gastroenterology and Nutrition. Arch Dis Child. 1990;65:909-11.

24. Caja S, Mäki M, Kaukinen K, Lindfors K. Antibodies in celiac disease: implications beyond diagnostics. Cell Mol Immunol. 2011;8:103-9.

25. Shiner M, Ballard J. Antigen-antibody reactions in journal mucosa in childhood celiac disease after gluten challenge. Lancet. 1972;1: 1202-5.

26. Savilahti E. Intestinal immunoglobulins in children with coeliac disease. Gut. 1972;13:958-64.

27. Jos J, Labbe F, Geny B, Griscelli C. Immunoelectron-microscopic localization of immunoglobulin A and secretory component in jejunal mucosa from children with coeliac disease. Scand J Immunol. 1979;9:441-50.

28. Lancaster-Smith M, Packer S, Kumar PJ, Harries JT. Immunological phenomena in the jejunum and serum after reintroduction of dietary gluten in children with treated coeliac disease. $\mathrm{J}$ Clin Pathol. 1976;29:592-7.

29. Kivel RM, Kearns DH, Liebowitz D. Significance of antibodies to dietary proteins in the serums of patients with nontropical sprue. $\mathrm{N}$ Engl J Med. 1964;271:769-72. 
30. Ladinser B, Rossipal E, Pittschieler K. Endomysium antibodies in coeliac disease: an improved method. Gut. 1994;35:776-8.

31. Salmaso C, Ocmant A, Pesce G, et al. Comparison of ELISA for tissue transglutaminase autoantibodies with antiendomysium antibodies in pediatric and adult patients with celiac disease. Allergy. 2001;56:544-7.

32. Chou R, Bougatsos C, Blazina I, Mackey K, Grusing S, Selph S. Screening for celiac disease: evidence report and systematic review for the US preventive services task force. JAMA. 2017;317:125868 .

33. Tursi A, Brandimarte G, Giorgetti GM. Prevalence of antitissue transglutaminase antibodies in different degrees of intestinal damage in celiac disease. J Clin Gastroenterol. 2003;36:219-21.

34. Husby S, Koletzko S, Korponay-Szabó IR, et al. European Society for Paediatric Gastroenterology, Hepatology, and Nutrition guidelines for the diagnosis of coeliac disease. J Pediatr Gastroenterol Nutr. 2012;54:136-60.

35. Rubio-Tapia A, Hill ID, Kelly CP, Calderwood AH, Murray JA. American College of Gastroenterology. ACG clinical guidelines: diagnosis and management of celiac disease. Am J Gastroenterol. 2013; 108:656-76.

36. Ludvigsson JF, Bai JC, Biagi F, et al. Diagnosis and management of adult coeliac disease: guidelines from the British Society of Gastroenterology. Gut. 2014;63:1210-28.

37. Bai JC, Fried M, Corazza GR, et al. World Gastroenterology Organisation global guidelines on celiac disease. J Clin Gastroenterol. 2013;47:121-6.

38. Al-Toma A, Volta U, Auricchio R, et al. European Society for the Study of Coeliac Disease (ESsCD) guideline for coeliac disease and other gluten-related disorders. United European Gastroenterol J. 2019;7:583-613.

39. Guidelines | Indian Council of Medical Research [Internet]. [cited 2018 Dec 30]. Available from: https://www.icmr.nic.in/guidelines? field select disease tid=92; downloaded on June 18 .

40. Fernández E, Riestra S, Rodrigo L, et al. Comparison of six human anti-transglutaminase ELISA-tests in the diagnosis of celiac disease in the Saharawi population. World J Gastroenterol. 2005;11:37626.

41. Porcelli B, Ferretti F, Vindigni C, Terzuoli L. Assessment of a test for the screening and diagnosis of celiac disease. J Clin Lab Anal. 2016;30:65-70.

42. Blackwell PJ, Hill PG, Holmes GK. Autoantibodies to human tissue transglutaminase: superior predictors of coeliac disease. Scand J Gastroenterol. 2002;37:1282-5.

43. Naiyer AJ, Hernandez L, Ciaccio EJ, et al. Comparison of commercially available serologic kits for the detection of celiac disease. J Clin Gastroenterol. 2009;43:225-32.

44. Singh P, Arora S, Lal S, Strand TA, Makharia GK. Risk of celiac disease in the first- and second-degree relatives of patients with celiac disease: a systematic review and meta-analysis. Am J Gastroenterol. 2015;110:1539-48.

45. Mishra A, Prakash S, Kaur G, et al. Prevalence of celiac disease among first-degree relatives of Indian celiac disease patients. Dig Liver Dis. 2016;48:255-9.

46. Castro-Antunes MM, Crovella S, Brandão LAC, Guimaraes RL, Motta MEFA, da Silva GAP. Frequency distribution of HLA DQ2 and DQ8 in celiac patients and first-degree relatives in Recife, northeastern Brazil. Clin Sao Paulo Braz. 2011;66:227-31.

47. Shergill S, Makharia GK. Awareness about celiac disease amongst physicians. Indian J Gastroenterol. 2017;36:327-9.

48. Catassi C, Fabiani E, Iacono G, et al. A prospective, double-blind, placebo-controlled trial to establish a safe gluten threshold for patients with celiac disease. Am J Clin Nutr. 2007;85:160-6.

49. Rajpoot P, Sharma A, Harikrishnan S, Baruah BJ, Ahuja V, Makharia GK. Adherence to gluten-free diet and barriers to adherence in patients with celiac disease. Indian J Gastroenterol. 2015;34:380-6.

50. Vohra P. India should worry about underdiagnosis and overdiagnosis of coeliac disease. BMJ. 2014;348:g2046.

Publisher's note Springer Nature remains neutral with regard to jurisdictional claims in published maps and institutional affiliations. 